\title{
Cl-Nd (Chlorine-Neodymium)
}

\section{H. Okamoto}

The Nd-Cl phase diagram shown in Fig. 1 was obtained by [1963Nov] by measuring cooling curves. Five compounds are shown in Fig. 1, but crystal structure data are available only for $\mathrm{NdCl}_{2}$ and $\mathrm{NdCl}_{3}$ [1973Lan] (Table 1). There must be at least three more intermediate compounds in this system on the $\mathrm{Nd}$-rich side of $\mathrm{NdCl}_{2}$ because of the invariant reactions observed at 818,808 , and $764{ }^{\circ} \mathrm{C}$. Invariant reactions at 1021 and $864{ }^{\circ} \mathrm{C}$ correspond to the melting point and the $\beta$ to $\alpha$ allotropic transformation temperature of $\mathrm{Nd}$, respectively.

\section{References}

1963Nov: G.I. Novikov and O.G. Polyachenok: Russ. J. Inorg. Chem., 1963, vol. 8 (5), pp. 545-50.

1973Lan: Landolt-Börnstein: III7a, Crystal Structure Data of Inorganic Compounds, Springer-Verlag, Berlin, 1973.

Table 1 Nd-Cl crystal structure data

\begin{tabular}{|c|c|c|c|c|c|}
\hline Phase & $\begin{array}{c}\text { Composition, } \\
\text { at.\% Cl }\end{array}$ & Pearson symbol & Space group & $\begin{array}{c}\text { Strukturbericht } \\
\text { designation }\end{array}$ & Prototype \\
\hline$(\beta \mathrm{Nd})$ & 0 & $c I 2$ & $\operatorname{Im} \overline{3} m$ & $A 2$ & W \\
\hline$(\alpha \mathrm{Nd})$ & 0 & $h P 4$ & $\mathrm{~Pb}_{3} / \mathrm{mmc}$ & $A 3^{\prime}$ & $\alpha \mathrm{La}$ \\
\hline $\mathrm{NdCl}_{2}$ & 66.7 & $o P 12$ & Pmnb & $C 23$ & $\mathrm{Co}_{2} \mathrm{Si}$ \\
\hline $\mathrm{Nd}_{4} \mathrm{Cl}_{9}$ & 69.2 & $\cdots$ & $\cdots$ & $\cdots$ & $\cdots$ \\
\hline $\mathrm{Nd}_{7} \mathrm{Cl}_{16}$ & 69.6 & $\cdots$ & $\cdots$ & $\cdots$ & $\cdots$ \\
\hline $\mathrm{Nd}_{3} \mathrm{Cl}_{7}$ & 70 & $\cdots$ & $\ldots$ & $\ldots$ & $\ldots$ \\
\hline $\mathrm{NdCl}_{3}$ & 75 & $h P 8$ & $P 6_{3} / m$ & $\cdots$ & $\mathrm{UCl}_{3}$ \\
\hline
\end{tabular}

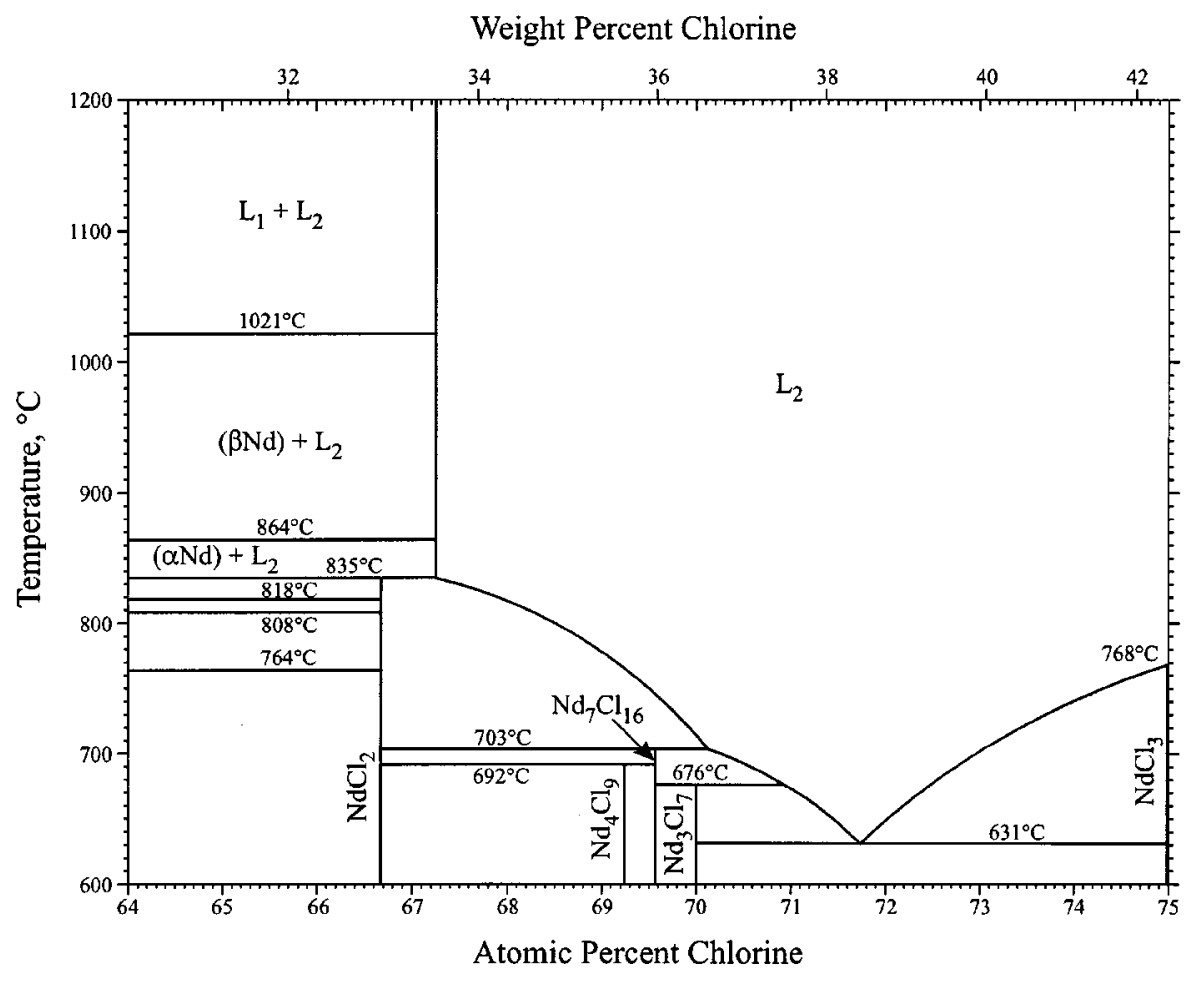

Fig. $1 \mathrm{Cl}-\mathrm{Nd}$ phase diagram 